\title{
A Symbolic Computation Approach to Parameterizing Controller for Polynomial Hamiltonian Systems
}

\author{
Zhong Cao ${ }^{1,2}$ and Xiaorong Hou ${ }^{1}$ \\ ${ }^{1}$ School of Energy Science and Engineering, University of Electronic Science and Technology of China, Chengdu, Sichuan 611731, China \\ ${ }^{2}$ School of Computer Science and Educational Software, Guangzhou University, Guangzhou, Guangdong 510006, China \\ Correspondence should be addressed to Xiaorong Hou; houxr@uestc.edu.cn
}

Received 2 April 2014; Accepted 10 June 2014; Published 26 June 2014

Academic Editor: Yingwei Zhang

Copyright (c) 2014 Z. Cao and X. Hou. This is an open access article distributed under the Creative Commons Attribution License, which permits unrestricted use, distribution, and reproduction in any medium, provided the original work is properly cited.

\begin{abstract}
This paper considers controller parameterization method of $H_{\infty}$ control for polynomial Hamiltonian systems (PHSs), which involves internal stability and external disturbance attenuation. The aims of this paper are to design a controller with parameters to insure that the systems are $H_{\infty}$ stable and propose an algorithm for solving parameters of the controller with symbolic computation. The proposed parameterization method avoids solving Hamilton-Jacobi-Isaacs equations, and thus the obtained controllers with parameters are relatively simple in form and easy in operation. Simulation with a numerical example shows that the controller is effective as it can optimize $H_{\infty}$ control by adjusting parameters. All these results are expected to be of use in the study of $H_{\infty}$ control for nonlinear systems with perturbations.
\end{abstract}

\section{Introduction}

A lot of control problems can be modeled as or transformed approximately to polynomial control systems, which are of great significance in the nonlinear theory. In recent years, a series of achievements have been obtained via symbolic computation and engineering applications [1-3]. The symbolic computation is an efficient and strong tool for polynomial control systems. Meanwhile, generalized Hamiltonian systems are such important nonlinear systems that they have been widely applied in engineering, physical and life science, and so forth. Many scholars studied generalized Hamiltonian systems in recent decades [4-6]. In control design, generalized Hamiltonian systems have good structural properties, which are clear physical expression, good structure and the Hamiltonian function in such a system can be used as good candidate of Lyapunov function. So we consider the polynomial Hamiltonian systems through combining their advantages for control theory. However, the current studies of polynomial Hamiltonian systems (PHSs) merely remain in mathematical theory $[7,8]$, and robust control and controller parameterization are hardly mentioned. This paper is concerned with $H_{\infty}$ control problems of such systems.
As we all know, the internal stability and the external disturbance attenuation are the basic constraints of control systems. However, it is critical to satisfy some desired control objectives in designing a practical control system. It is a sophisticated and efficient way to find the parameterized controller to solve complex control problems, namely, the $H_{\infty}$ control, which can satisfy the additional design objectives. Therefore, controller parameterization is a fundamental problem in the control theory and has aroused considerable attention in recent decades [9-19]. Lu and Doyle [10] and Isidori and Astolfi [11] proposed a family of nonlinear $H_{\infty}$ controllers via output feedback. Astolfi [12] presented a family of nonlinear state-feedback controllers, in which the system state and the external disturbance are measurable. Yung et al. $[13,14]$ extended the state-space formulas and presented a family of $H_{\infty}$ state-feedback controllers for $n$-dimensional nonlinear system. Fu et al. $[15,16]$ proposed a family of reliable nonlinear $H_{\infty}$ controllers via solving the HamiltonJacobi-Isaacs (HJI) inequality (or equations). Xu and $\mathrm{Hou}$ $[17,18]$ studied the generalized Hamiltonian system and proposed a family of parameterized controllers in $H_{\infty}$ control and adaptive control. Furthermore, the controllers have been applied to synchronous generators with steam valve in [19]. 
The controllers obtained in [10-16] are intended to solve a class of HJI inequalities (or equations), which have actually imposed a considerable difficulty. To obtain the parameterized controllers, [17-19] avoided solving HJI inequalities (or equations) applying good structure and clear physical expression of general Hamiltonian systems. However, there are so many restrictions in the process of obtaining a controller in which the form would be very complex even it could not be achieved.

In order to avoid solving HJI inequalities (or equations) and obtain a parameterized controller, this paper presents a novel, straightforward, and convenient method to parameterizing controller for PHSs and gives an algorithm for solving parameters of the controller by symbolic computation. The main results of the paper are as follows.

(1) A controller with parameters is obtained via the approach to parameterizing controller for PHSs. Because the Hamiltonian function can be used to build a Lyapunov function of a dissipative Hamiltonian system, the approach obtained here avoids solving HJI inequalities (or equations). Moreover, the obtained controller with parameters has a simpler form than the controller obtained in [17].

(2) Parameters ranges are obtained via an algorithm based on symbolic computation. The controller with parameters is effective to $H_{\infty}$ control and it can optimize $H_{\infty}$ control performance of systems by adjusting parameters.

The remainder of this paper is organized as follows. In Section 2, the problem of $H_{\infty}$ control for PHSs is formulated. The main contribution of this paper is then given in Section 3, in which a controller with parameters and an algorithm for solving parameters are provided, respectively. We present a numerical example for illustrating effectiveness and feasibility of $H_{\infty}$ controller in Section 4 and conclusions follow in Section 5 .

\section{Problem Formulation and Preliminaries}

Consider the following polynomial Hamiltonian system with dissipation [20]:

$$
\begin{gathered}
\dot{x}=[J(x)-R(x)] \nabla H(x)+g_{1}(x) u+g_{2}(x) \omega, \\
y=g_{2}^{T}(x) \nabla H(x), \\
z=h(x) g_{1}^{T}(x) \nabla H(x),
\end{gathered}
$$

where $x \in \mathbb{R}^{n}$ is the state vector; $u \in \mathbb{R}^{m}$ is the controller with parameters; $\omega \in \mathbb{R}^{s}$ is the disturbances; $J(x)$ is a skewsymmetric matrix; $R(x)$ is a positive semidefinite matrix; $g_{1}(x) \in \mathbb{R}^{n \times m}$ and $g_{2}(x) \in \mathbb{R}^{n \times s}$ are sufficiently smooth functions; $y \in \mathbb{R}^{p}$ is the output; $z \in \mathbb{R}^{q}$ is the penalty; $h(x)$ is a weighting matrix; $H(x)$ is the Hamiltonian function which has a local minimum at the equilibrium $x_{0}$ of system, and $\nabla H(x)=(\partial H / \partial x)(x)$. The Hamiltonian function $H(x)$ must be a positive semidefinite polynomial and the following assumption holds.
Assumption 1. $H(x) \in C^{2}$ and the Hessian matrix Hess $(H$ $\left.\left(x_{0}\right)\right)>0$.

Remark 2. Note that $H_{i}(x)$ has a local minimum at the equilibrium $x_{0}$ of system (1). It is straightforward that in Assumption $1, H_{i}(x) \in C^{2}$ guarantees the existence of $\operatorname{Hess}\left(H_{i}(x)\right)$ and Hess $\left(H_{i}\left(x_{0}\right)\right)>0$ guarantees that $H_{i}(x)$ is strict convex on some neighborhood of equilibrium $x_{0}$.

The problem considered in this paper is to propose an approach to parameterizing controller for systems (1), which can be described as follows: given a disturbance attenuation level $\gamma>0$, we can obtain a controller with parameters of the form $u=\alpha(x)\left(\alpha\left(x_{0}\right)=0\right)$ such that the $L_{2}$ gain of the closedloop system (from $\omega$ to $z$ ) is bounded by $\gamma$, and the closedloop system is locally asymptotically stable when $\omega=0$.

In the end, we give a definition and a lemma required in the next section.

Definition 3 (see [20]). System (1) is called zero-energygradient (ZEG) observable with respect to $y$ if $y(t)=0$ and $\omega(t)=0, \forall t \geq 0$, implies $\nabla H(x(t))=0, \forall t \geq 0$; system (1) is called ZEG detectable with respect to $y$ if $y(t)=0$ and $\omega(t)=0, \forall t \geq 0$, implies $\lim _{t \rightarrow \infty} \nabla H(x(t))=0$; system (1) is called generalized ZEG observable (detectable) if $y(t)=0$, $z(t)=0, \omega(t)=0, \forall t \geq 0$, implies $\nabla H(x(t))=0, \forall t \geq 0$ $\left(\lim _{t \rightarrow \infty} \nabla H(x(t))=0\right)$.

Lemma 4 (see [21]). Consider a nonlinear system

$$
\begin{gathered}
\dot{x}=f(x)+g(x) \omega, \quad f\left(x_{0}\right)=0, \\
z=h(x),
\end{gathered}
$$

where $x \in \mathbb{R}^{n}$ is the state vector, $\omega \in \mathbb{R}^{s}$ is the disturbances, and $z \in \mathbb{R}^{q}$ is the penalty. If there exists the function $V(x) \geq$ $0\left(V\left(x_{0}\right)=0\right)$ such that HJI inequality

$$
\begin{aligned}
& \left(\frac{\partial V}{\partial x}\right)^{T} f(x)+\frac{1}{2 \gamma^{2}}\left(\frac{\partial V}{\partial x}\right)^{T} g(x) g(x)^{T} \frac{\partial V}{\partial x} \\
& +\frac{1}{2} h(x)^{T} h(x) \leq 0
\end{aligned}
$$

holds, it is implied that the $L_{2}$ gain of the closed-loop system (2) (from $\omega$ to $z$ ) is bounded by $\gamma(\gamma>0)$; that is,

$$
\int_{0}^{T}\|z\|^{2} d t \leq \int_{0}^{T} \gamma^{2}\|\omega\|^{2} d t
$$

\section{Main Results}

In this section, we propose an $H_{\infty}$ controller with parameters for system (1) and an algorithm for solving parameters. The parameterization methods suggest a framework to solve the $H_{\infty}$ control problem of polynomial Hamiltonian systems. 


\subsection{Parameterizing Controller}

Theorem 5. Suppose Assumption 1 holds and also suppose system (1) is generalized ZEG detectable (when $\omega=0$ ) and

$$
\begin{gathered}
R(x)+\frac{1}{2 \gamma^{2}}\left(g_{1} g_{1}^{T}-g_{2} g_{2}^{T}\right) \geq 0, \\
\nabla H^{T} g_{1} K(x) \leq 0
\end{gathered}
$$

hold simultaneously (i.e., the matrix $R(x)+\left(1 / 2 \gamma^{2}\right)\left(g_{1} g_{1}^{T}-\right.$ $\left.g_{2} g_{2}^{T}\right)$ is positive semidefinite and $\nabla H^{T} g_{1} K(x)$ is negative semidefinite). Then, $H_{\infty}$ control of system (1) can be realized by the following controller:

$$
u=-\frac{1}{2}\left(h^{T} h+\frac{1}{\gamma^{2}} I_{m}\right) g_{1}^{T} \nabla H+K(x)
$$

where $K(x) \in \mathbb{R}^{m \times 1}$ is item containing parameters, which is part of the controller parameterization, and $I_{m}$ is an $m \times m$ unit matrix.

Proof. Consider the candidate Lyapunov function $V(x)=$ $H(x)-c \geq 0\left(c=H\left(x_{0}\right)\right)$. From Lemma 4 and controller (7), we have

$$
\begin{aligned}
\left(\frac{\partial V}{\partial x}\right)^{T} f(x)+\frac{1}{2 \gamma^{2}}\left(\frac{\partial V}{\partial x}\right)^{T} g g^{T}\left(\frac{\partial V}{\partial x}\right)+\frac{1}{2} z^{T} z \\
=-\nabla H^{T} R(x) \nabla H+\nabla H^{T} g_{1} u+\frac{1}{2 \gamma^{2}} \nabla H^{T} g_{2} g_{2}^{T} \nabla H \\
\quad+\frac{1}{2} z^{T} z \\
=-\nabla H^{T}\left(R(x)+\frac{1}{2} g_{1} h^{T} h g_{1}^{T}+\frac{1}{2 \gamma^{2}} g_{1} g_{1}^{T}\right) \nabla H \\
\quad+\nabla H^{T} g_{1} K(x)+\frac{1}{2 \gamma^{2}} \nabla H^{T} g_{2} g_{2}^{T} \nabla H \\
\quad+\frac{1}{2} \nabla H g_{1} h^{T} h g_{1}^{T} \nabla H \\
=-\nabla H^{T}\left(R(x)+\frac{1}{2 \gamma^{2}}\left(g_{1} g_{1}^{T}-g_{2} g_{2}^{T}\right)\right) \nabla H \\
+\nabla H^{T} g_{1} K(x) \leq 0
\end{aligned}
$$

which implies that the $L_{2}$ gain of the closed-loop system (1) controlled by controller (7) (from $\omega$ to $z$ ) is bounded by $\gamma$. Next, we prove that the closed-loop system is asymptotically stable at $x_{0}$, when $\omega=0$. From system (1), controller (7), and $\omega=0$, it follows that

$$
\begin{aligned}
\dot{V}(x)= & \left(\frac{\partial V}{\partial x}\right)^{T}[J(x)-R(x)]\left(\frac{\partial V}{\partial x}\right)+\left(\frac{\partial V}{\partial x}\right)^{T} g_{1} u \\
= & -\nabla H^{T} R(x) \nabla H+\nabla H^{T} g_{1} \\
& \times\left[-\frac{1}{2}\left(h^{T} h+\frac{1}{\gamma^{2}}\right) g_{1}^{T} \nabla H+K(x)\right] \\
= & -\nabla H^{T}\left(R(x)+\frac{1}{2 \gamma^{2}}\left(g_{1} g_{1}^{T}-g_{2} g_{2}^{T}\right)\right) \nabla H \\
& -\frac{1}{2} \nabla H^{T} g_{1} h^{T} h g_{1}^{T} \nabla H \\
& -\frac{1}{2 \gamma^{2}} \nabla H^{T} g_{2} g_{2}^{T} \nabla H+\nabla H^{T} g_{1} K(x) \\
= & -\nabla H^{T}\left(R(x)+\frac{1}{2 \gamma^{2}}\left(g_{1} g_{1}^{T}-g_{2} g_{2}^{T}\right)\right) \nabla H \\
& -\frac{1}{2}\left\|h g_{1}^{T} \nabla H\right\|^{2} \\
& -\frac{1}{2 \gamma^{2}}\left\|g_{2}^{T} \nabla H\right\|^{2}+\nabla H^{T} g_{1} K(x) \leq 0 .
\end{aligned}
$$

Hence, the closed-loop system converges to the largest invariant set, which is contained in

$$
\begin{aligned}
S & =\{x: \dot{V}(x)=0\} \\
& \subset\left\{x: y=g_{2}^{T} \nabla H=0, z=h(x) g_{1}^{T}(x) \nabla H=0, \forall t \geq 0\right\} .
\end{aligned}
$$

From Assumption 1 and the fact that system (1) is generalized ZEG detectable, we obtain

$$
\lim _{t \rightarrow \infty} \nabla H(x(t))=\nabla H\left(\lim _{t \rightarrow \infty} x(t)\right)=0 .
$$

On the other hand, since $H(x)$ has a local minimum at the equilibrium $x_{0}$, it shows $\nabla H\left(x_{0}\right)=0$. From Assumption 1, there exists a neighborhood of $x_{0}$, in which $\nabla H\left(\lim _{t \rightarrow \infty} x\right.$ $(t))=0$ implies $\lim _{t \rightarrow \infty} x(t)=x_{0}$. Therefore, from (11), it follows that the trajectory of $\dot{V}(x)=0$ is strict convex on the equilibrium $x_{0}$. According to the LaSalle invariant principle, the closed-loop system (1) controlled by controller (7) is locally asymptotically stable at $x_{0}$. This completes the proof.

Remark 6. (1) As compared with the controller proposed in [13-19], the $H_{\infty}$ controller (7) has a much simpler form and is easier to realize.

(2) Parameters ranges of polynomial vector $K(x)$ can be obtained by solving condition (6).

(3) Wang et al. $[22,23]$ presented the dissipative Hamiltonian realization method for general nonlinear systems that can be transformed to PHSs. So the controller obtained in this paper can be applied to general nonlinear systems. 
3.2. Solving Parameters Algorithm. From condition (5), when $R(x)+\left(1 / 2 \gamma^{2}\right)\left(g_{1} g_{1}^{T}-g_{2} g_{2}^{T}\right)=0$, we can obtain the $\gamma^{*}$. Let $\gamma \geq \gamma^{*}$ such that condition (5) holds. Then we propose an algorithm to find parameters ranges of controller (7) via solving the parameters of $K(x)$ in condition (6). The algorithm now proceeds as follows.

S1. Set $K(x)=\left[\begin{array}{llll}K_{1}(x) & K_{2}(x) & \cdots & K_{m}(x)\end{array}\right]^{T}$ and suppose a positive integer $r$, which is the degree of polynomial vector $K(x)$. Write $K_{i}(x)=\sum_{j=1, r}^{j=l} a_{i j} p_{r}(x)$, where $l=\sum_{r} c(n+r-1, r), p_{r}(x)=\prod_{i=1}^{n} x_{i}^{r_{i}}$ and $n$ is the number of state variable.

S2. Let $S=-\nabla H^{T} g_{1} K(x)$.

S3. The influence of high order items can be ignored because this paper considers locally asymptotically stable for system. Choose all terms of $\operatorname{deg}(S) \geq 3$ and $\operatorname{deg}(S)=1$ from $S$ and let the coefficients of these terms be zero. So obtain a set of equations $A$.

S3.1. Observe equations $A$. When the right-hand side is only one item with parameters and the left-hand side is zero, let these parameters be zero and substitute them into $A$. Then obtain simplified equations $A^{\prime}$.

S3.2. Obtain a set of parameters solution $U_{1}$ via solving $A^{\prime}$ by using cylindrical algebraic decompositions (CAD) algorithm [24].

S3.3. Substitute $U_{1}$ into $S$ and obtain a new polynomial $S$, which is a quadratic form.

S4. Rewrite $S$ as coefficient matrix $M$, and all principal minors of $M$ must be positive semidefinite [25]. Choose all principal minors of $M$ and obtain inequalities $B$.

S4.1. Observe inequalities $B$. Let some parameters be zero and substitute them into $B$. Then obtain the simplified inequalities $B^{\prime}$.

S4.2. Obtain a set of parameters solution $U_{2}$ via solving $B^{\prime}$ by using CAD algorithm.

S5. Let $U=U_{1} \cup U_{2}$ and substitute $U$ into controller (7), and obtain the polynomial parameterized controller. This completes the algorithm.

Remark 7. (1) The algorithm starts from $r=1$ normally.

(2) The CAD algorithm is given by Semi-Algebraic-SetTools of Regular-Chains in Maple 16.

(3) It is merely to simplify computation that we let some parameters be zero before using CAD algorithm. However, these parameters are not necessarily zero. So the set of parameters solution obtained by the algorithm is a subset of solutions.

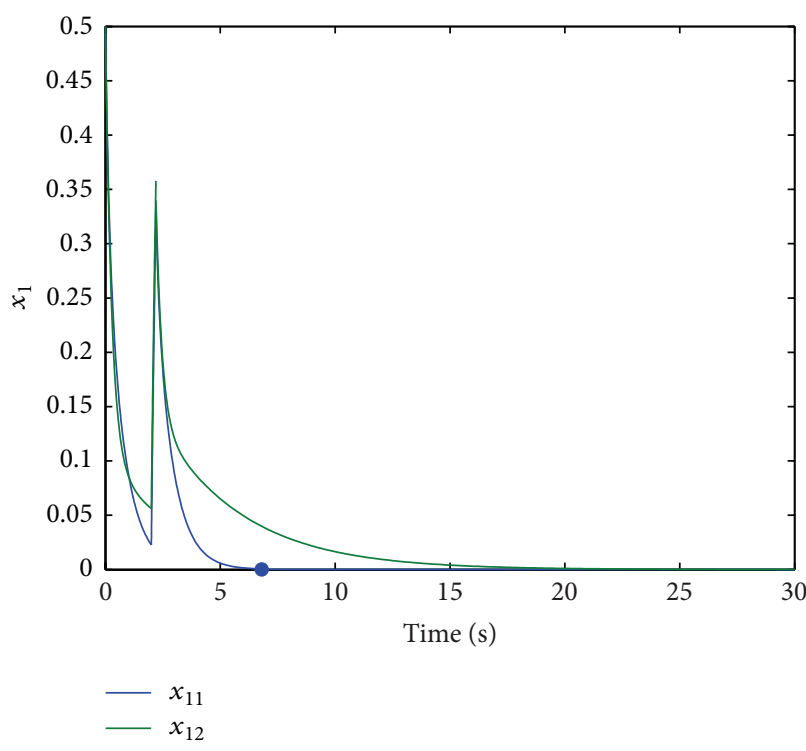

FIGURE 1: Swing curves of $x_{1}$.

\section{Numerical Experiments}

Consider a polynomial Hamiltonian system with dissipation (1)

$$
\begin{gathered}
J(x)=\left[\begin{array}{cccc}
0 & 1 & 0 & 0 \\
-1 & 0 & 0 & x_{2} \\
0 & 0 & 0 & 0 \\
0 & -x_{2} & 0 & 0
\end{array}\right], \quad g_{1}=\left[\begin{array}{lll}
0 & 0 & 0 \\
1 & 0 & 0 \\
0 & 1 & 0 \\
0 & 0 & 1
\end{array}\right], \\
g_{2}=\left[\begin{array}{lll}
1 & 0 & 0 \\
0 & 0 & 0 \\
0 & 1 & 0 \\
0 & 0 & 1
\end{array}\right], \quad R(x)=\operatorname{Diag}\{4,0,2,2\}, \\
H(x)=\frac{1}{2}\left(x_{1}^{2}+2 x_{1} x_{2}+2 x_{2}^{2}+2 x_{3}^{2}+2 x_{3} x_{4}+x_{4}^{2}\right) .
\end{gathered}
$$

4.1. Controller Design and Solving Parameters. From system (12), it is easy to get

$$
\operatorname{Hess}\left(H\left(x_{0}\right)\right)=\left[\begin{array}{llll}
1 & 1 & 0 & 0 \\
1 & 2 & 0 & 0 \\
0 & 0 & 2 & 1 \\
0 & 0 & 1 & 1
\end{array}\right]>0 .
$$

So Assumption 1 holds.

Then, we check that condition (5) holds for all $x$ and given $\gamma$. From system (12), we have

$$
R(x)+\frac{1}{2 \gamma^{2}}\left(g_{1} g_{1}^{T}-g_{2} g_{2}^{T}\right)=\operatorname{Diag}\left\{4-\frac{1}{2 \gamma^{2}}, \frac{1}{2 \gamma^{2}}, 2,2\right\} .
$$

Let $\gamma^{*}=\sqrt{2} / 4$. To ensure that condition (5) holds, the following statement should be satisfied

$$
\gamma \geq \gamma^{*} .
$$


Next, we consider condition (6) such that system (12) satisfies robustness in $H_{\infty}$ control. Set the candidate Lyapunov function $V(x)=H(x)$. It follows from controller (7) that

$$
u=\left[\begin{array}{l}
u_{1} \\
u_{2} \\
u_{3}
\end{array}\right]=-\frac{1}{2}\left[h^{T} h+\frac{1}{\gamma^{2}} I_{m}\right] g_{1}^{T} \nabla H+K,
$$

where $K=\left[\begin{array}{lll}K_{1} & K_{2} & K_{3}\end{array}\right]^{T}$.

We know $n=4$ in system (12) and let $r=1$. We have

$$
\begin{aligned}
& K_{1}=a_{1} x_{1}+a_{2} x_{2}+a_{3} x_{3}+a_{4} x_{4}, \\
& K_{2}=b_{1} x_{1}+b_{2} x_{2}+b_{3} x_{3}+b_{4} x_{4}, \\
& K_{3}=c_{1} x_{1}+c_{2} x_{2}+c_{3} x_{3}+c_{4} x_{4},
\end{aligned}
$$

where $a_{i}, b_{i}, c_{i}, i=1,2,3,4$ are the parameters.

$$
M=\left[\begin{array}{cccc}
-2 a_{1} & -2 a_{1}-a_{2} & -2 b_{1}-a_{3}-c_{1} & -c_{1}-a_{4}-b_{1} \\
-2 a_{1}-a_{2} & -4 a_{2} & -c_{2}-2 a_{3}-2 b_{2} & -b_{2}-2 a_{4}-c_{2} \\
-2 b_{1}-a_{3}-c_{1} & -c_{2}-2 a_{3}-2 b_{2} & -4 b_{3}-2 c_{3} & -b_{3}-2 b_{4}-c_{3}-c_{4} \\
-c_{1}-a_{4}-b_{1} & -b_{2}-2 a_{4}-c_{2} & -b_{3}-2 b_{4}-c_{3}-c_{4} & -2 b_{4}-2 c_{4}
\end{array}\right] .
$$
$\left[\begin{array}{llll}x_{1}+x_{2} & x_{1}+2 x_{2} & 2 x_{3}+x_{4} & x_{3}+x_{4}\end{array}\right]^{T}$.

Let $S=-\nabla H^{T} g_{1} K$; we have

$$
\begin{aligned}
S= & -a_{1} x_{1}^{2}-\left(2 a_{1}+a_{2}\right) x_{1} x_{2}-\left(2 b_{1}+a_{3}+c_{1}\right) x_{1} x_{3} \\
& -\left(c_{1}+a_{4}+b_{1}\right) x_{1} x_{4}-2 a_{2} x_{2}^{2}-\left(c_{2}+2 a_{3}+2 b_{2}\right) x_{2} x_{3} \\
& -\left(b_{2}+2 a_{4}+c_{2}\right) x_{2} x_{4}-\left(2 b_{3}+c_{3}\right) x_{3}^{2} \\
& -\left(b_{3}+2 b_{4}+c_{3}+c_{4}\right) x_{3} x_{4}-\left(b_{4}+c_{4}\right) x_{4}^{2}
\end{aligned}
$$

$S$ is a quadratic form and can be rewritten as a coefficient matrix (multiply constant 2 for simplifying computation),
All principal minors of $M$ must be positive semidefinite. We have inequalities $B$ from $M$. From $B$, we can easy obtain that $a_{1} \leq 0, a_{2} \leq 0$, and $a_{2}=2 a_{1}$. Substitute $U_{1}=\left\{a_{3}=\right.$ $\left.0, c_{4}=0, b_{1}=0, b_{2}=0, c_{1}=0, c_{2}=0\right\}$ into inequalities $B$ to simplify computation; we obtain simplified inequalities $B^{\prime}$. Solving inequalities $B^{\prime}$ by using CAD algorithm, we obtain a series of sets. Choose some sets, which satisfy inequalities $B^{\prime}$, and organize them. We have

$$
U=\left\{a_{1} \leq 0, c_{3}=-b_{3}, c_{4}=-2 b_{4}, b_{3} \leq 0, b_{4} \geq 0\right\} \cup U_{1} .
$$

Substitute $U$ into controller (7),

$$
u=-\frac{1}{2}\left[h^{T} h+\frac{1}{\gamma^{2}} I_{m}\right]\left[\begin{array}{c}
x_{1}+2 x_{2} \\
2 x_{3}+x_{4} \\
x_{3}+x_{4}
\end{array}\right]+\left[\begin{array}{c}
a_{1} x_{1}+2 a_{1} x_{2} \\
b_{3} x_{3}+b_{4} x_{4} \\
-b_{3} x_{3}-2 b_{4} x_{4}
\end{array}\right]
$$

where $a_{1} \leq 0, b_{3} \leq 0, b_{4} \geq 0$. So we have the controller with parameters for system (12). The controller (21) has a rather simple form.

4.2. Simulations and Results. In order to evaluate the robustness of the controller (21), we set the parameters of system (12) as $\gamma=1, h=\operatorname{Diag}\{1,1,1\}$, and the parameters of controller as $a_{1}=-1, b_{3}=-1, b_{4}=1$. We obtain the following controller:

$$
u=\left[\begin{array}{c}
-2 x_{1}-4 x_{2} \\
-3 x_{3} \\
-3 x_{4}
\end{array}\right]
$$

Suppose that $x(0)=(0.05,0,0,-0.05)^{T}$ is the preassigned operating point of system (12); we impose an external disturbance $\omega=[2,2,2]^{T}$ on system (12) during the time period
From system (12), we obtain that $\nabla H(x)=$

$2 \sim 2.2 \mathrm{~s}$. In order to evaluate the robust performance of controller (22), we compare it with the saturated controller proposed in [26]. The parameters of the saturated controller are chosen as follows: $K_{1}=0.01, K_{2}=0.01, K_{3}=0.01$ and $L_{1}=0.05, L_{2}=0.05, L_{3}=0.05$.

The simulation results are shown in Figures 1, 2, 3, and 4, where $\left(x_{11}, x_{21}, x_{31}, x_{41}\right)^{T}$ is the response of the state $x$ under controller (22) and $\left(x_{12}, x_{22}, x_{32}, x_{42}\right)^{T}$ is the response of the state $x$ under saturated controller in [26]. From Figures 1-4, we can clearly see that under controller (22), it takes only 6.8 seconds for the system to return back to the equilibrium point (circle point in figures), while under saturated controller, it takes about 24 seconds. The simulation shows that controller (22) is much more efficient and feasible and has stronger robustness than saturated controller.

In order to evaluate the robustness optimization of the system by adjusting the parameters of controller (21), we choose the parameters of system (12) as $\gamma=1, h=\operatorname{Diag}\{1,1$, $1\}$ and the parameters of controller as $a_{1}=-100, b_{3}=-100$, $b_{4}=100$. We have the following controller:

$$
u=\left[\begin{array}{c}
-101 x_{1}-202 x_{2} \\
-102 x_{3}+99 x_{4} \\
99 x_{3}-201 x_{4}
\end{array}\right]
$$

Suppose that $x(0)=(0.05,0,0,-0.05)^{T}$ is the preassigned operating point of system (12); we impose an external disturbance $\omega=[2,2,2]^{T}$ on system (12) during the time period 2 2.2 s. In Figure 5, $\left(x_{1}, x_{2}, x_{3}, x_{4}\right)^{T}$ is the response of the state $x$ under controller (22), and in Figure $6,\left(x_{1}, x_{2}, x_{3}, x_{4}\right)^{T}$ is the response of the state $x$ under controller (23).

From Figure 5, we can clearly see that under controller (22), it takes 6.8 seconds for the system to return back to the equilibrium point (circle point in figures), while under controller (23), it takes about 4.8 seconds in Figure 6. The 


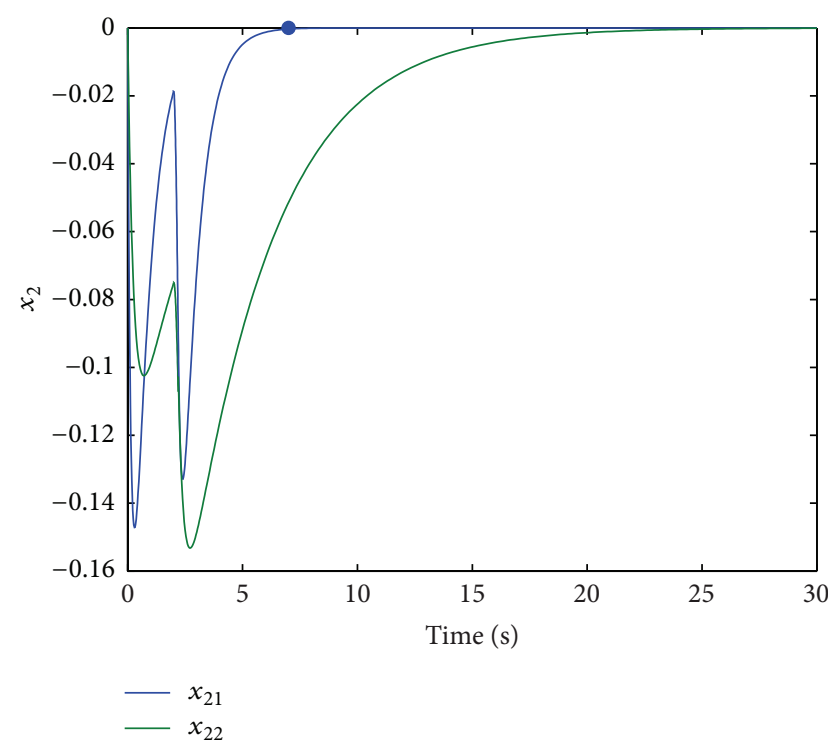

FIGURE 2: Swing curves of $x_{2}$.

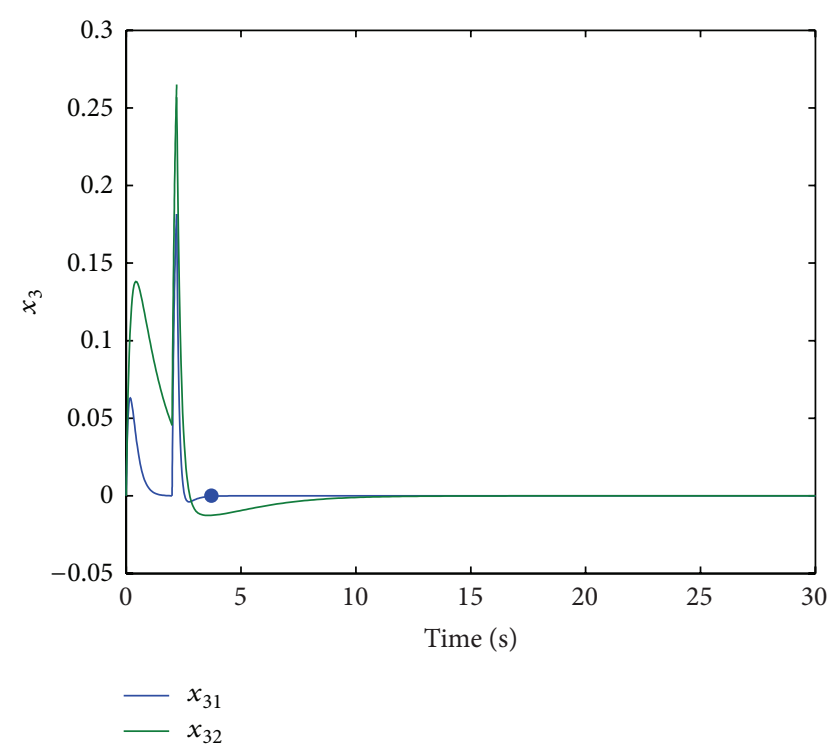

FIGURE 3: Swing curves of $x_{3}$.

time of state vectors $x_{3}$ and $x_{4}$ back to the equilibrium point is much shorter in Figure 5 than in Figure 6. The simulation shows that controller (21) can effectively optimize the robustness of system by adjusting parameters.

\section{Conclusion}

In this paper, an approach to parameterizing controller for polynomial Hamiltonian systems has been considered. A controller with parameters has been obtained using Hamiltonian function method and an algorithm for solving

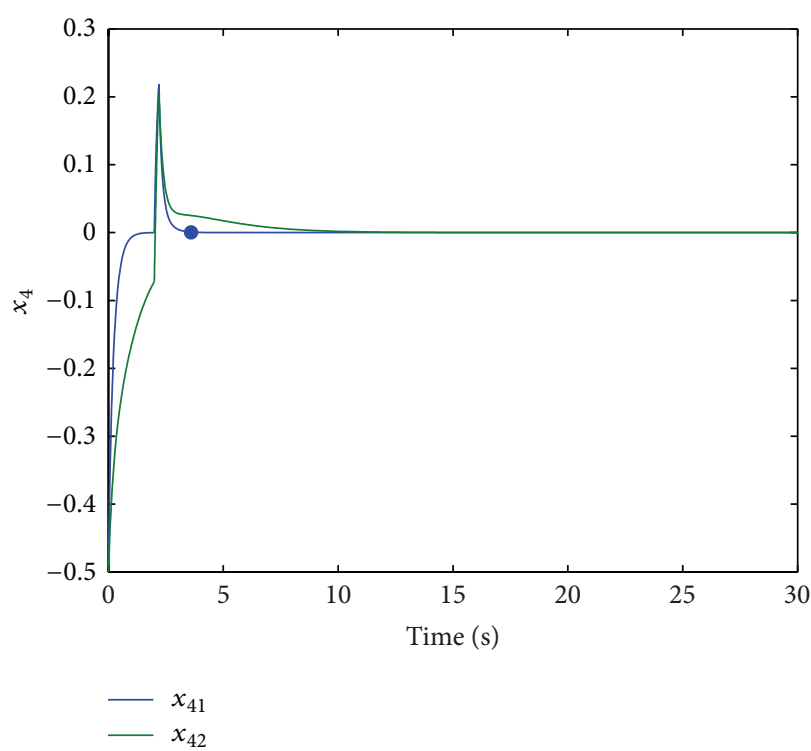

Figure 4: Swing curves of $x_{4}$.

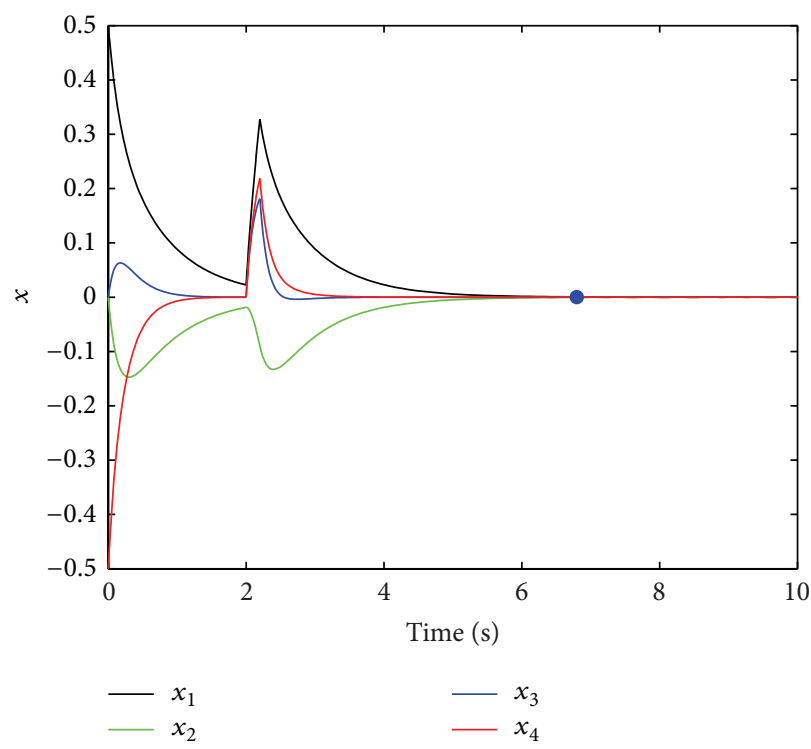

FIGURE 5: Swing curves of $x_{1} \sim x_{4}$.

parameters of the controller has been proposed with symbolic computation. The proposed parameterization method avoids solving Hamilton-Jacobi-Isaacs equations and thus the obtained controllers with parameters are easier as compared to some existing ones. The numerical experiment and simulations show that the controller is efficient in $H_{\infty}$ control; meanwhile the controller can optimize the robustness of the system by adjusting parameters. The approach proposed in this paper is also applicable to general nonlinear systems, which should be transformed to the polynomial Hamiltonian systems through the available dissipative Hamiltonian realization methods. 


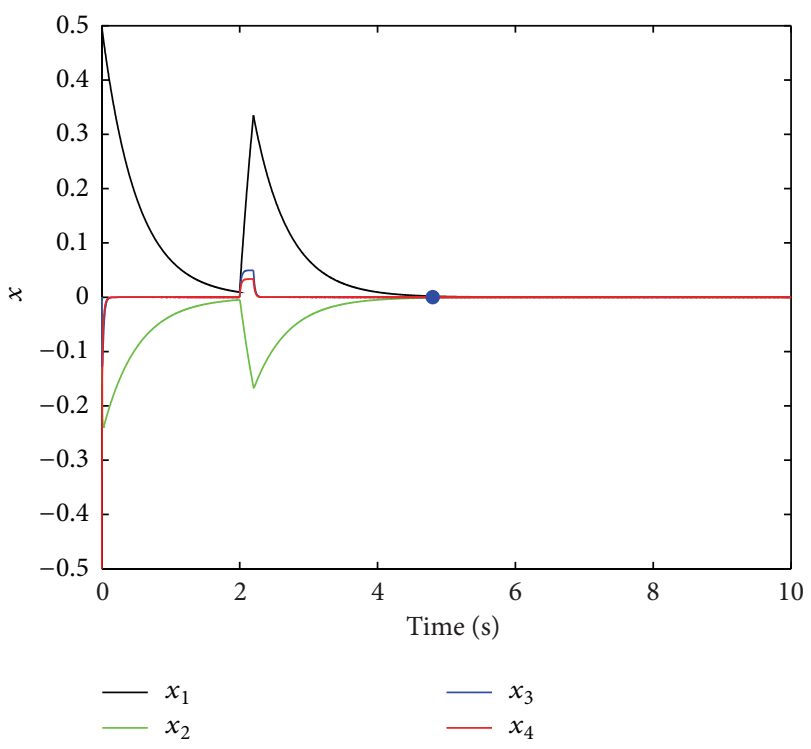

FIGURE 6: Swing curves of $x_{1} \sim x_{4}$.

\section{Conflict of Interests}

The authors declare that there is no conflict of interests regarding the publication of this paper.

\section{Acknowledgment}

This work is supported by the National Natural Science Foundation of China (nos. 61074189 and 61374001).

\section{References}

[1] Y. Huang, Y. Shang, and W. Yuan, "Symbolic computation and the extended hyperbolic function method for constructing exact traveling solutions of nonlinear PDEs," Journal of Applied Mathematics, vol. 2012, Article ID 716719, 19 pages, 2012.

[2] J. F. Navarro, "The asymptotic expansion method via symbolic computation," Journal of Applied Mathematics, vol. 2012, Article ID 201340, 24 pages, 2012.

[3] H. Deng and J. Wu, "Approximate bisimulation and optimization of software programs based on symbolic-numeric computation," Mathematical Problems in Engineering, vol. 2013, Article ID 421926, 19 pages, 2013.

[4] R. Ortega, A. van der Schaft, B. Maschke, and G. Escobar, "Interconnection and damping assignment passivity-based control of port-controlled Hamiltonian systems," Automatica, vol. 38, no. 4, pp. 585-596, 2002.

[5] Y. Wang, S. S. Ge, and D. Cheng, "Observer and observer-based $H_{\infty}$ control of generalized Hamiltonian systems," Science in China F, vol. 48, no. 2, pp. 211-224, 2005.

[6] I. G. Polushin, "Stabilization of invariant tori in Hamiltonian systems under persistently acting disturbances," International Journal of Robust and Nonlinear Control, vol. 11, no. 3, pp. 253265, 2001.

[7] H. Zhou, W. Xu, S. Li, and Y. Zhang, "On the number of limit cycles of a cubic polynomials Hamiltonian system under quintic perturbation," Applied Mathematics and Computation, vol. 190, no. 1, pp. 490-499, 2007.
[8] F. Iavernaro and D. Trigiante, "High-order symmetric schemes for the energy conservation of polynomial Hamiltonian problems," Journal of Numerical Analysis, Industrial and Applied Mathematics, vol. 4, no. 1-2, pp. 87-101, 2009.

[9] J. Gadewadikar, F. L. Lewis, L. Xie, V. Kucera, and M. AbuKhalaf, "Parameterization of all stabilizing Ho static statefeedback gains: application to output-feedback design," Automatica, vol. 43, no. 9, pp. 1597-1604, 2007.

[10] W.-M. Lu and J. C. Doyle, " $\mathscr{H}_{\infty}$ control of nonlinear systems via output feedback: controller parameterization," IEEE Transactions on Automatic Control, vol. 39, no. 12, pp. 2517-2521, 1994.

[11] A. Isidori and A. Astolfi, "Disturbance attenuation and $H_{\infty}$ control via measurement feedback in nonlinear systems," IEEE Transactions on Automatic Control, vol. 37, no. 9, pp. 1283-1293, 1992.

[12] A. Astolfi, "Parameterization of output feedback controller that satisfy an norm bound," in Proceedings of the 2nd European Control Conference, pp. 74-78, Groningen, The Netherlands, 1993.

[13] C. Yung, Y. Lin, and F. Yeh, "A family of nonlinear $\mathrm{H}_{\infty}$ output feedback controllers," Institute of Electrical and Electronics Engineers. Transactions on Automatic Control, vol. 41, no. 2, pp. 232-236, 1996.

[14] C. F. Yung, J. L. Wu, and T. T. Lee, "Parameterization of nonlinear $H_{\infty}$ state-feedback controllers," Automatica, vol. 33, no. 8, pp. 1587-1590, 1997.

[15] Y. Fu, Z. Tian, and S. Shi, "A family of reliable nonlinear Ho state-feedback controllers," Control Theory and Applications, vol. 18, no. 3, pp. 447-452, 2001.

[16] Y. S. Fu, Z. H. Tian, and S. J. Shi, "A family of reliable state-feedback controllers for nonlinear systems with strictly redundant actuators: the full information case," Journal of Systems Science and Systems Engineering, vol. 10, no. 3, pp. 350358, 2001.

[17] S. Xu and X. Hou, "A family of $H_{\mathrm{\infty}}$ controllers for dissipative Hamiltonian systems," International Journal of Robust and Nonlinear Control, vol. 22, no. 11, pp. 1258-1269, 2012.

[18] S. Xu and X. Hou, "A family of adaptive $\mathrm{H}_{\infty}$ controllers with full information for dissipative Hamiltonian systems," International Journal of Automation and Computing, vol. 8, no. 2, pp. 209-214, 2011.

[19] S. Xu and X. Hou, "A family of robust adaptive excitation controllers for synchronous generators with steam valve via Hamiltonian function method," Journal of Control Theory and Applications, vol. 10, no. 1, pp. 11-18, 2012.

[20] A. van der Schaft and A. J. Schaft, L2-Gain and Passivity in Nonlinear Control, Springer, Berlin, Germany, 1999.

[21] T. Shen, Control Theory and its Applications, Tsinghua University Press, Beijing, China, 1996.

[22] Y. Wang, D. Cheng, C. Li, and Y. Ge, "Dissipative Hamiltonian realization and energy-based $L_{2}$-disturbance attenuation control of multimachine power systems," IEEE Transactions on Automatic Control, vol. 48, no. 8, pp. 1428-1433, 2003.

[23] Y. Wang, D. Cheng, and S. S. Ge, "Approximate dissipative Hamiltonian realization and construction of local Lyapunov functions," Systems \& Control Letters, vol. 56, no. 2, pp. 141-149, 2007.

[24] F. B. Caviness and R. J. Johnson, Quantifier Elimination and Cylindrical Algebraic Decomposition, Springer, New York, NY, USA, 1998. 
[25] K. N. Swamy, "Sylvester's criterion for positive-semidefinite matrices," IEEE Transactions on Automatic Control, vol. 18, no. 3, p. 306, 1973.

[26] Z. Xi, G. Feng, D. Cheng, and Q. Lu, "Nonlinear decentralized saturated controller design for power systems," IEEE Transactions on Control Systems Technology, vol. 11, no. 4, pp. 539-547, 2003. 


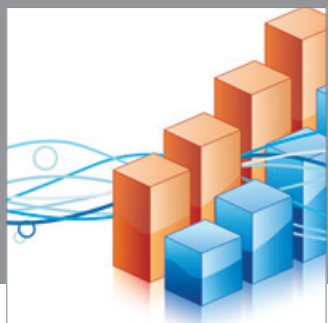

Advances in

Operations Research

mansans

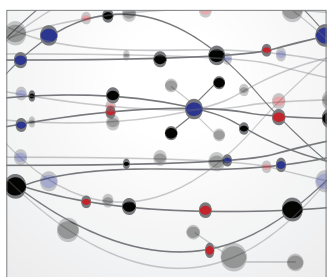

The Scientific World Journal
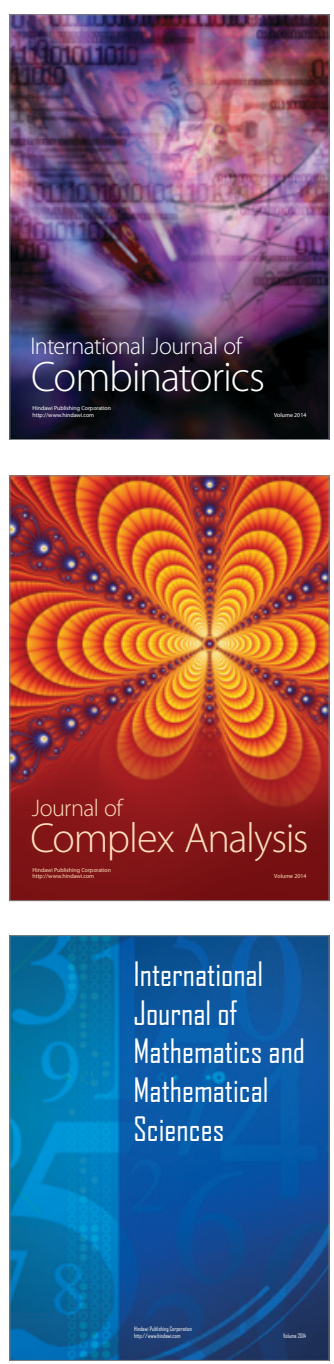
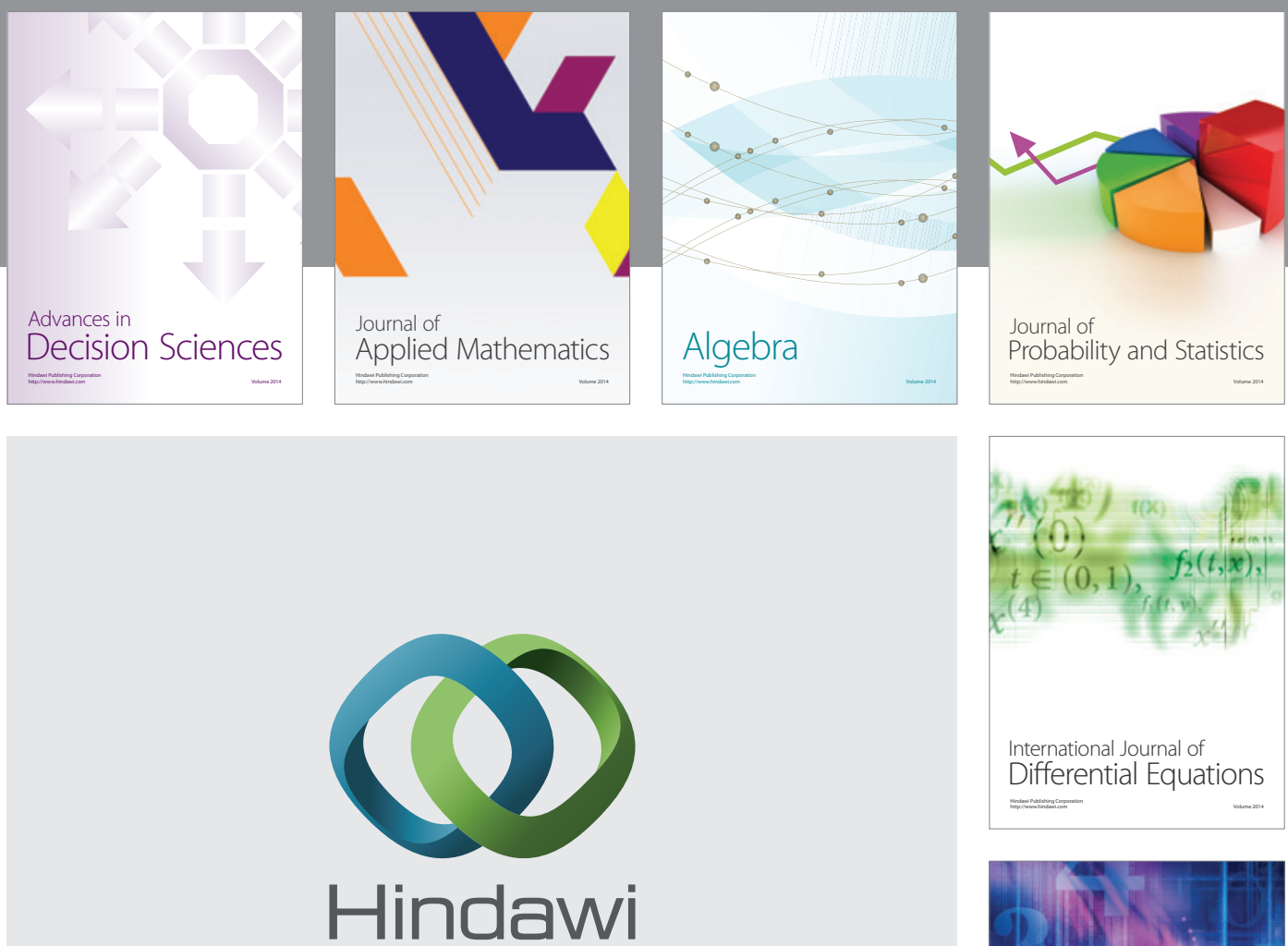

Submit your manuscripts at http://www.hindawi.com
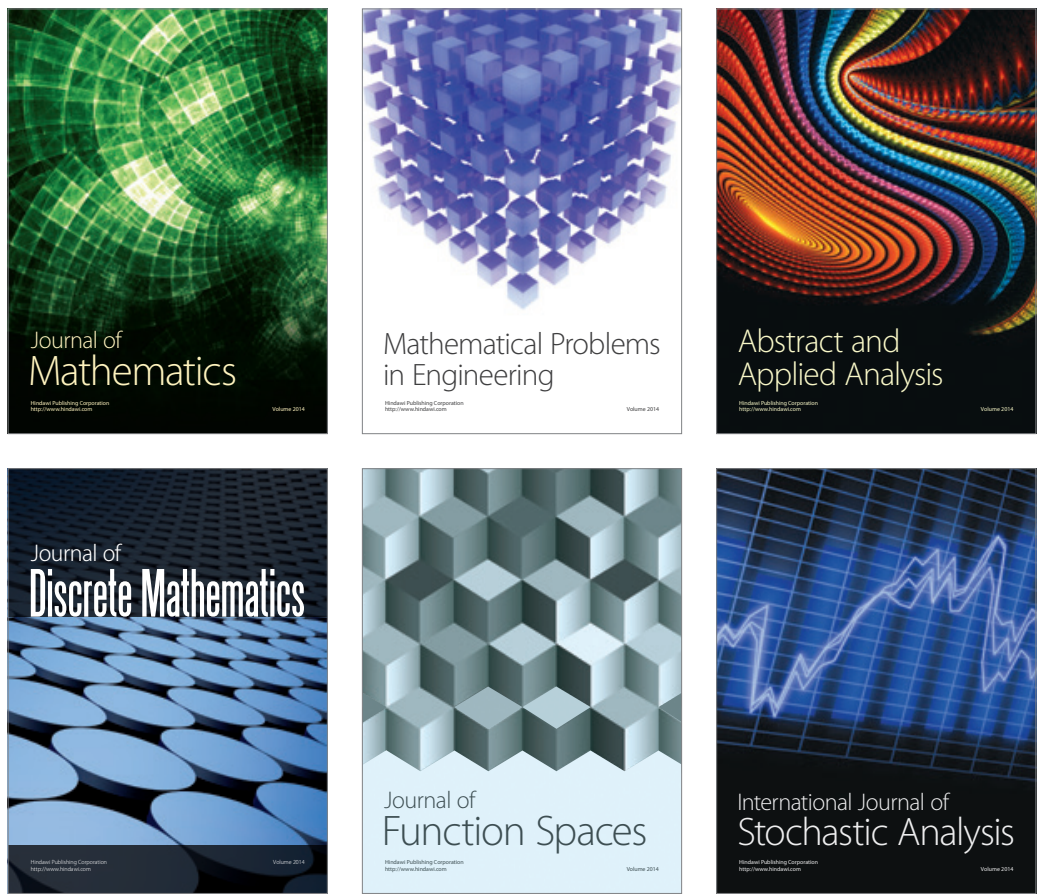

Journal of

Function Spaces

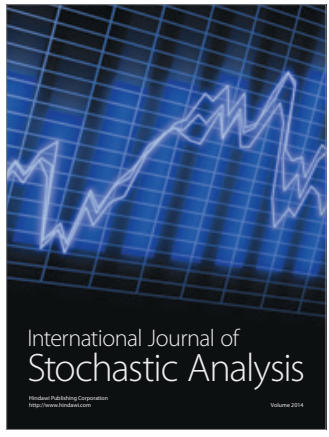

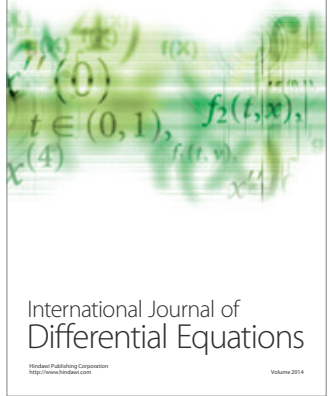
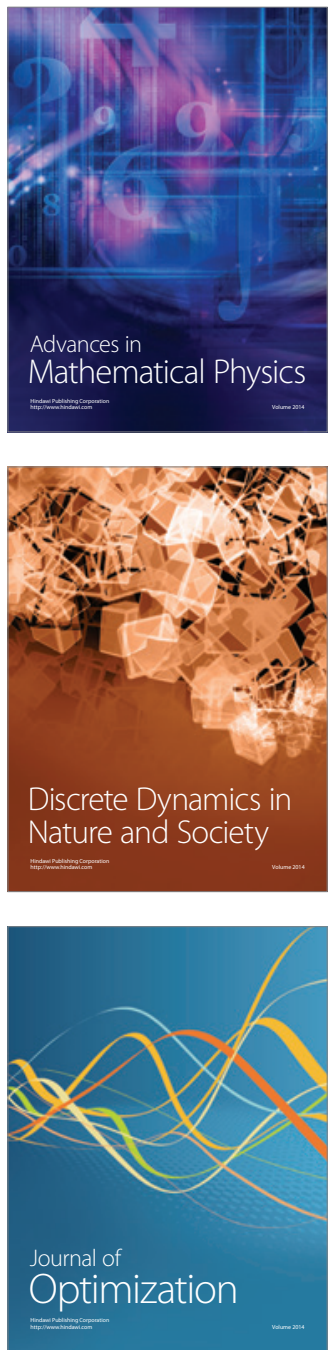